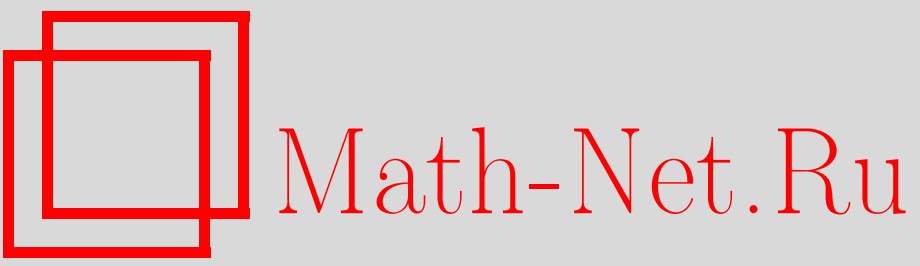

А. Г. Витушкин, А. А. Гончар, М. В. Самохин, В. М. Тихомиров, П. Л. Ульянов, В. П. Хавин, В. Я. Эйдерман, Семен Яковлевич Хавинсон (некролог), УМН, 2004, том 59, выпуск 4, 186-192

DOI: https://doi.org/10.4213/rm762

Использование Общероссийского математического портала Math-Net.Ru подразумевает, что вы прочитали и согласны с пользовательским соглашением

http: //www.mathnet.ru/rus/agreement

Параметры загрузки:

IP: 3.95 .254 .165

26 апреля 2023 г., 14:19:21 


\section{СЕМЕН ЯКОВЛЕВИЧ ХАВИНСОН}

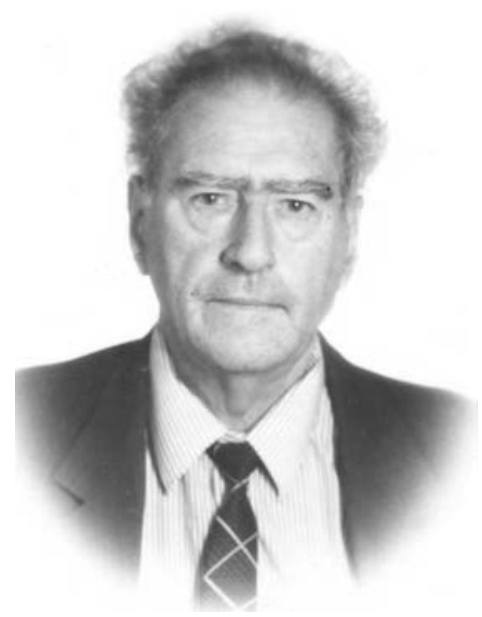

Заниматься научной работой Семен Яковлевич начал еще в студенческие годы: его первая научная публикация [1] появилась в 1949 г. С 1949 по 1953 г. он обучается в заочной аспирантуре мехмата МГУ под руководством профессора А.И. Маркушевича. В 1953 г. С. Я. Хавинсон успешно защищает кандидатскую диссертацию, а в 1962 г. и докторскую.

Известность С. Я. Хавинсону принес уже первьй цикл работ, посвященных исследованию широкого круга экстремальных задач в различных классах аналитических функций. С. Я. Хавинсон разработал общий подход к решению экстремальных задач, основанный на соотношениях двойственности. Исходным пунктом явилась двойственность между задачей об экстремуме некоторых линейных функционалов и задачей о наилучшем приближении в сопряженном пространстве, установленная в классических работах М.Г. Крейна (1938) и С. М. Никольского (1946) и являющаяся следствием теоремы Хана-Банаха. Экстремальными задачами частного характера, в которых возникают подобные соотношения двойственности, занимались многие замечательные ученые. Э. Ландау (1913), О. Сас $(1916,1929)$, Ф. Рисс (1920), Я. Л. Геронимус (1937-1948), Г. М. Голузин (1946), А. Макинтайр и В. Рогозинский (1950) рассматривали различные классы аналитических функций в круге. Значительно позже началось изучение экстремальных задач в многосвязных областях: Г. Грунский $(1940,1942)$, Л. Альфорс (1947), П. Гарабедян (1950), Р. Робинсон (1944), М. Хейнс (1940). Но соотношения двойственности, полученные в работах перечисленных выше авторов, не только не являлись инструментом исследования экстремальных функций, но, наоборот, выводились из специальных свойств этих функций. С. Я. Хавинсон был первым, кто начал (с 1949 г. - см. [1], [2]) развивать и использовать метод двойственности применительно к экстремальньм задачам теории аналитических функций. Взаимосвязанное рассмотрение двойственных задач способствовало более глубокому изучению каждой из них. При этом резултаты предшествующих авторов гармонично вписались в общую теорию, разработанную С.Я. Хавинсоном, и получили там свое уточнение и развитие. Указанньй подход позволил, например, решить вопрос о единственности экстремальных элементов, исследовать их устройство 
и свойства, а в ряде случаев и явно выписать соответствующие экстремальные функции [3], [8], [11], [32], [37]. С.Я. Хавинсон распространил свою теорию и на классы функций с различными дополнительными условиями (например, с ограничениями на рост в области их определения и с дополнительными ограничениями на рост на некотором подмножестве области определения; типичной является известная задача Мию (1924)). Были найдены двойственные соотношения для таких задач [18], [38]; см. также обзор [24].

Яркой демонстрацией эффективности развитого метода двойственности явилась теория аппроксимационных процессов, в которых требуется не только установить полноту заданной системы функций в нормированном пространсте $X$, но и оценить коэффициенты аппроксимирующих полиномов. Некоторые специалные случаи подобной аппроксимации рассматривались Дейвисом и Фань-Цзы (Ки Фан) (1957), но общая теория была построена С.Я. Хавинсоном [14], [18]-[20], [30], [31], [42]. Нужный критерий был выведен им из двойственных соотношений, найденных в [16], [23] для "возмущенной" задачи аппроксимации, к которой приводит указанная вьше постановка. В качестве следствий получаются разнообразные эффектные и важные приложения. Например, классические теоремы Лаврентьева, Мюнца и Вейерштрасса дополняются точными оценками коэффициентов аппроксимирующих многочленов [26], [31], [34]. Развитие и дальнейшие применения этой теории имеются в [35], [41].

Задача установления полноты системы функций в $X$ часто сводится к таким теоремам единственности для аналитических функций $f(z)$ с определенным ограничением на рост, в которых заключение $f(z) \equiv 0$ вьводится из равенств $f\left(z_{n}\right)=0$, где $\left\{z_{n}\right\}$ - достаточно "массивная" последовательность точек, не сгущающаяся внутри области. С.Я. Хавинсон показал, что вопросы полноты с учетом величин коэффициентов связаны с более "деликатными" теоремами единственности, в которых тождество $f(z) \equiv 0$ вытекает из того, что $f(z)$ лишь достаточно мала в точках последователшности. Им был получен один из первых резултатов такого типа [16], [23], явившийся стимулом для целого ряда исследований различных авторов.

Аппарат, разработанньй С.Я. Хавинсоном при решении экстремальных и аппроксимационных задач, был успешно применен им для исследования классических проблем устранения особенностей. В [39], [40] излагается теория, охватывающая разнообразные классы функций. В частности, получено окончательное решение задачи об устранимых множествах для класса В.И. Смирнова. Особое место занимает задача об аналитической емкости $\gamma(F)$ множеств и ее модификациях. Как писал позднее Семен Яковлевич [53], в исследованиях экстремальных задач типа задачи об аналитической емкости можно с определенной долей условности выделить два направления: "метрическое" и "аналитическое". В первом из них $\gamma(F)$ сравнивается с более удобными геометрическими и метрическими характеристиками. Во втором, "аналитическом" направлении, к которому преимущественно относятся исследования С.Я. Хавинсона, рассматривается структура самих экстремальных задач для различных классов функций. Здесь возникают следующие вопросы: какие свойства данного класса аналитических функций отображаются соответствующей экстремальной величиной ("емкостью")? Если эта величина равна нулю, следует ли, что данный класс функций тривиален? Каковы специфические свойства аппроксимационных процессов, возникающих на множествах с нулевой "емкостью"? Разумеется, указанные направления тесно связаны. Так, резултаты С.Я. Хавинсона об аналитической емкости компактов общего вида позволили внести весомый вклад [21] в доказательство знаменитой "геометрической" гипотезы Данжуа (1909). С.Я. Хавинсон был первым, кто начал изучать [12], [13], [15], [22] задачи об аналитической емкости в произвольных областях. Были установлены характеристические свойства экстремальной функции (функции Альфорса), ее единственность, изучены аналогичные вопросы для классов функций, отличных от $B^{1}$, получен ряд резултатов о совместной нетривиальности различных классов функций. Исследованы вопросы представимости функций интегралами типа Коши (потенциалами Коши), а также связь между аналитической емкостью и распределением масс и, в частности, экстремальное распределение. Получены важные теоремы об аппроксимации на множествах аналитической емкости нуль с учетом величин коэффициентов аппроксимирующих полиномов [15], ряд других существенных результатов.

Использование соотношений двойственности для классов аналитических функций в многосвязных областях стало возможным благодаря глубокому изучению различных свойств этих классов, предпринятому в работах С.Я. Хавинсона и в серии его совместных работ с Г.Ц. Тумаркиньм [4]-[7], [9], [10], [17]. Прежде всего отметим результаты о факторизации 
функций, обобщающие классическую факторизацию Смирнова-Неванлинны на многосвязные области. Основная трудность здесь состояла в том, что замена ядра Пуассона (вещественной части ядра Шварца) ядром Грина в многосвязной области немедленно приводит к появлению многозначности.

Резултаты описанных выше исследований были изложены С.Я. Хавинсоном в монографии "Экстремальные свойства и множества устранимых особенностей аналитических функций" (660 c.), представленной для публикации в издательство "Наука". Но сложности с книгоизданием в те годы и, прежде всего, субъективизм при отборе рукописей для публикации привели к тому, что эта замечательная книга не была принята к печати. Чтобы огромный труд не пропал, Семен Яковлевич был вынужден искусственно разбить монографию на части и опубликовать их (частично за свои средства) в издательстве МИСИ как пособия для слушателей факултета повышения квалификации преподавателей [36]-[40]. Две из этих частей ([37], [38]) были переведены и изданы Американским математическим обществом [44].

Развивая теорию фак торизации в многосвязных областях, С. Я. Хавинсон рассмотрел [45]-[47] однозначные аналитические функции на компактных римановых поверхностях с краем, состоящим из аналитических контуров. Им был предложен новый оригиналшный способ устранения многозначности при построении вариантов ядра Шварца и произведения Бляшке. Даже в случае плоской многосвязной области этот метод предоставляет бо́льшие возможности, чем известные ранее.

С.Я. Хавинсон плодотворно занимался и задачами представления или аппроксимации функций нескольких переменных линейными суперпозициями функций меньшего числа переменных [27], [43], [48]-[52]. Данное направление начало развиваться благодаря работам А. Г. Витушкина, А.Н. Колмогорова, В.И. Арнольда и др. по решению тринадцатой проблемы Гилберта. Среди многих исследованных здесь вопросов отметим проблемы, связанные с переходом от функций двух переменных к случаю бо́лшшего числа переменных. Такое обобщение не только потребовало преодоления ряда существенных трудностей, но и привело к открытию новых эффектов. Итогом этих исследований стала монографиия [52] (см. также [59]).

Нельзя не отметить и замечательный цикл недавних работ С. Я. Хавинсона по изучению модифокикацй аналитической емкости и связанных с ними задач аппроксимации, ставший, к несчастью, последним. В [54] теория аппроксимации с учетом величин коэффицциентов распространена на случай, когда приближение ведется с наперед заданной скоростью. В статье [53], носящей одновременно и обзорный, и исследовательский характер, рассматриваются классы функций, представимые более общим аналитическим аппаратом, чем потенциалы Коши, а именно, так называемыми суммами Голубева, содержащими производные различных порядков потенциалов Коши. Полученные результаты обобщают известные ранее критерии о представимости потенциалами Коши. Далее излагается общая схема исследования экстремальных задач типа задачи об аналитической емкости. Двойственная задача оказьвается задачей об аппроксимации на множествах нулевой емкости с учетом величин коэффициентов. При этом суммы Голубева с разными классами мер (комплексными, действительными и положительными) приводят к различным модификациям аналитической емкости. Наиболее подробно рассматривается случай емкости Коши (термин введен С.Я. Хавинсоном в $[25]): \gamma_{C}(F)=\sup \left|\int_{F} d \mu\right|$, где верхняя грань берется по всем комплексным мерам с носителями на компакте $F$, для которых модуль потенциала Коши не превосходит единицы в $\mathbb{C} \backslash F$. Особенно специфической оказалась задача аппроксимации, соответствующая положительным мерам в суммах Голубева. Убедительным свидетельством глубины и плодотворности разработанной С. Я. Хавинсоном теории явилась также фундаментальная работа [58]. Вначале устанавливаются двойственные соотношения для емкостей $\gamma, \gamma_{C}$ и их модификаций. Затем теория аппроксимации с учетом величин коэффициентов обобщается на случай, когда аппроксимация ведется элементами некоторого клина, а не линейного подпространства, как это всегда делалось прежде. В [57], [58] получены соотношения двойственности для такой аппроксимации. Они порождают целое семейство новых емкостных величин, являющихся естественными обобщениями известных ранее.

Работы [53], [58], с одной стороны, подводят итог сделанному ранее, а с другой - демонстрируют огромньй потенциал разработанных С. Я. Хавинсоном методов, возможность дальнейшего глубокого развития его теории. Труды С. Я. Хавинсона давно получили мировое признание; его результаты заняли достойное место в математической литературе. Укажем, например, известные монограффии П. Кусиса "Введение в теорию пространств $H^{p}$ ”, М.: Мир, 1984, и Дж. Гарнетта "Ограниченные аналитические функции”, М.: Мир, 1984. 
Семен Яковлевич был таким ярким, неординарным человеком, какие встречаются редко. Его талант находил проявления в самых разнообразных видах деятельности. Нечасто блестящий, активно работающий математик оказьвается и прекрасным руководителем. В течение 32 лет с 1964 по 1996 г. - С. Я. Хавинсон возглавлял кафедру высшей математики МИСИ (ныне МГСУ - Московский государственньй строителшный университет), а проработал в МИСИ-МГСУ с 1956 г. до последних дней жизни. Кафедра являлась одной из самых болшших кафедр математики в СССР; в 80-е годы численность ее сотрудников превышала 80 человек. Такие коллективы обычно делятся на две, а то и на три части. Здесь этого не произошло: все хотели работать под руководством Семена Яковлевича! Обаяние его личности, независимость и ясность мышления, глубокая порядочность и интеллигентность притягивали людей. Его авторитет был исключительно высок. Во многом благодаря С.Я. Хавинсону на кафедре сложилась атмосфера творчества, доброжелателшности, взаимовыручки, ощущение коллектива близких по духу людей. Концепция С.Я. Хавинсона преподавания математики будущим инженерам легла в основу созданной под его руководством системы обучения, по существу, действующей и поныне. Мощный творческий заряд Семена Яковлевича оказьвал огромное влияние на научную жизнь кафедры. Были (и далеко не единичные) случаи, когда преподаватели, переходившие в МГСУ из других институтов, получали здесь как бы второе дыхание, вовлеченные в атмосферу научного поиска. Так было, например, с инициированными им плодотворными исследованиями по аппроксимации линейными суперпозициями; статья [51] - лишь одна из серии вьполненных здесь работ и совместно с С.Я. Хавинсоном, и отдельно. Более подробная библиография этих работ имеется в [50], [52]. Возглавляемые им научные коллективы математиков поддерживались грантами Международного научного фонда (Сороса), РФФИ, Госкомвуза РФ. Ряд ученых кафедры Семена Яковлевич вырастил сам. Под его руководством подготовлено и защищено десять кандидатских диссертаций. Для математика, проработавшего почти всю жизнь в техническом вузе, не имеющем студентов-математиков, это весьма и весьма высокий резултат. Двое из его учеников (М. В. Самохин и В. Я. Эйдерман) стали докторами физико-математических наук. М. В. Самохин с 1996 г. заведует кафедрой.

С.Я. Хавинсон сам являлся блестящим преподавателем. В течение 11 лет он читал лекции по высшей математике по Централшному телевидению, являлся членом научно-методического совета Минвуза СССР. Многие годы С.Я. Хавинсон работал на факултете повьшения квалификации преподавателей математики (ФПКП), являя слушателям яркий образец лекторского искусства. Его учебник [33] по интегральному исчислению - одно из лучших пособий по этой теме для студентов ВТУЗов. Отметим также брошюры [28], [29], написанные совместно с М. И. Сканави, учебное пособие [56], увлекательный факультативньй курс [55] для студентов-первокурсников. При этом указанные ниже труды С. Я. Хавинсона - лишь неболшшая часть его научного и методического наследия, содержащего около 180 работ. Список первых 55 из них (за 1949-1966 годы) имеется в [П1], [П2].

С.Я. Хавинсон награжден почетным знаком "За отличные успехи в работе" в области высшего образования и знаком "Почетньй работник высшего образования России", являлся Почетным профессором МГСУ.

Вместе с тем Семен Яковлевич был чутким, отзывчивым человеком, всегда готовым помочь, и помогавший очень многим. За его спиной кафедра чувствовала себя защищенной. Он был широким эрудитом не только в математике. Тонкий знаток поэзии, он мог часами по памяти читать стихи. И писал сам; его поэзия была глубокой и пронзительной.

Неоправданные жертвы, которые несли наука и образование в последние 15 лет, вызывали у него чувство горечи. Но Семен Яковлевич продолжал самоотверженно работать. Страшньй удар сразил С. Я. Хавинсона в расцвете его многогранного таланта. Многое из задуманного сделать не удалось. На столе осталась почти завершенная рукопись ... .

Когда уходят такие люди, мир становится беднее. Неправда, что незаменимых нет: заменить Семена Яковлевича невозможно. Масштаб его личности навсегда останется ориентиром, определяющим и уровень научных исследований, и уровень преподавания, и отношение к делу и друг к другу.

А.Г. Витушкин, А.А. Гончар, М.В. Самохин, В. М. Тихомиров, П.Л. Ульянов, В. П. Хавин, В. Я. Эйдерман 


\section{ЦИТИРОВАННЫЕ РАБОТЫ С. Я. ХАВИНСОНА}

[1] Об одной экстремальной задаче теории аналитических функций // УМН. 1949. Т. 4. № 4. C. $158-159$

[2] О некоторых экстремальных задачах теории аналитических функций // Учен. зап. МГУ. Сер. матем. 1951. Т. 148. № 4. С. 133-143

[3] Экстремальные задачи для некоторых классов аналитических функций в конечно-связных областях // Матем. сб. 1955. Т. 36 (78). № 3. С. 445-478

[4] О стирании особенностей аналитических функций одного класса (класса $D$ ) // УМН. 1957. Т. 12. № 4. С. 193-199 (совм. с Г. Ц. Тумаркиным)

[5] Аналитические функции в многосвязных областях класса В. И. Смирнова (класса $S$ ) // Изв. АН СССР. Сер. матем. 1958. Т. 22. № 3. С. 379-386 (совм. с Г. Ц. Тумаркиным)

[6] О существовании в многосвязных областях однозначных аналитических функций с заданным модулем граничных значений // Изв. АН СССР. Сер. матем. 1958. Т. 22. № 4. С. 543-562 (совм. с Г.Ц. Тумаркиным)

[7] Условия представимости гармонической функции формулой Грина в многосвязной области // Матем. сб. 1958. Т. 44 (86). № 2. С. 225-234 (совм. с Г. Ц. Тумаркиным)

[8] Исследование свойств экстремальных функций с помощью соотношений двойственности в экстремальных задачах для классов аналитических функций в многосвязных областях // Матем. сб. 1958. Т. 46 (88). № 2. С. 195-228 (совм. с Г. Ц. Тумаркиным)

[9] Классы аналитических функций в многосвязных областях, представимые по формулам Коши и Грина // УМН. 1958. Т. 13. № 2. С. 215-221 (совм. с Г. Ц. Тумаркиным)

[10] О теореме разложения для аналитических функций класса $E_{p}$ в многосвязных областях // УМН. 1958. Т. 13. № 2. С. 223-228 (совм. с Г. Ц. Тумаркиным)

[11] О единственности функций наилучшего приближения в метрике пространства $L_{1} / /$ Изв. АН СССР. Сер. матем. 1958. Т. 22. № 2. С. 243-270

[12] Об аналитической емкости плоских множеств, о некоторых классах аналитических функций и об экстремальной функции в лемме Шварца для произвольных областей // Докл. АН CCCP. 1959. T. 128. № 5. C. 896-898

[13] Аналитическая емкость множеств и распределения масс // Докл. АН СССР. 1959. Т. 128. № 6. C. 1129-1131

[14] Об одном классе экстремальных задач для полиномов // Докл. АН СССР. 1960. Т. 130. № 5. C. $997-1000$

[15] Об аппроксимации на множествах аналитической емкости нуль // Докл. АН СССР. 1960. T. 131. № 1. C. 44-46

[16] Об экстремалшных задачах для функций, удовлетворяющих дополнительным условиям внутри области, и применении этих задач к вопросам аппроксимации // Докл. АН СССР. 1960. T. 135. № 2. C. $270-273$

[17] Классы аналитических функций в многосвязных областях // Исследования по современным проблемам теории функций комплексного переменного. М.: Физматгиз, 1960. С. 45-77 (совм. с Г. Ц. Тумаркиным)

[18] Об аппроксимации с учетом величин коэффициентов аппроксимирующих агрегатов // Труды МИАН. 1961. Т. 60. С. 304-324

[19] О двух классах экстремальных задач для полиномов и моментов // Изв. АН СССР. Сер. матем. 1961. Т. 25. № 4. С. 557-590

[20] Некоторые вопросы полноты систем // Докл. АН СССР. 1961. Т. 137. № 4. С. 793-796

[21] Некоторые оценки аналитической емкости // Докл. АН СССР. 1961. Т. 138. № 4. С. 789-792 (совм. с В. П. Хавиным)

[22] Об аналитической емкости множеств, совместной нетривиальности различных классов аналитических функций и лемме Шварца в произвольных областях // Матем. сб. 1961. Т. 54 (96). № 1 . C. $3-50$

[23] Теория экстремальных задач для ограниченных аналитических функций, удовлетворяющих дополнительным условиям внутри области // УМН. 1963. Т. 18. № 2. С. 25-98

[24] Аналитические функции ограниченного вида // Математический анализ. 1963. (Итоги науки). М.: ВИНИТИ, 1965. С. 5-80

[25] О представлении и приближении функций на редких множествах / Современные проблемы теории аналитических функций. М.: Наука, 1966. С. 314-318 
[26] Допустимые величины коэффициентов многочленов при равномерной аппроксимации непрерьвных функций // Матем. заметки. 1969. Т. 6. № 5. С. 619-625

[27] Чебышевская теорема для приближения функций двух переменных суммами $\varphi(x)+\psi(y)$ // Изв. АН СССР. Сер. матем. 1969. Т. 33. № 3. С. 650-666

[28] Основы векторного анализа (конспект лекций). Часть I. М.: Высшая школа, 1971 (совм. с М.И. Сканави)

[29] Теория поля и ее приложения к гидромеханике (конспект лекций). Часть II. М.: Высшая школа, 1971 (совм. с М. И. Сканави)

[30] Некоторые теоремы о приближении с учетом величин коэффициентов аппроксимирующих функций // Докл. АН СССР. 1971. Т. 196. №6. С. 1283-1286

[31] О понятии полноты, учитьвающем величины коэффициентов аппроксимирующих полиномов // Изв. АН АрмССР. Матем. 1971. Т. 6. № 2-3. С. 221-234

[32] О представлении экстремальных функций в классах $E_{q}$ через функции Грина и Неймана // Матем. заметки. 1974. Т. 16. № 5. С. 707-716

[33] Лекции по интегральному исчислению. М.: Высшая школа, 1976

[34] О коэффициентах многочленов в аппроксимационной теореме Вейерштрасса // Матем. заметки. 1977. Т. 22. № 2. С. 269-276 (совм. с О. А. Мурадян)

[35] Об одном классе точных неравенств для полиномов, моментов и аналитических функций // Сиб. матем. журн. 1981. Т. 22. №6. С. 188-207

[36] Факторизация аналитических функций в конечносвязных областях. Учебное пособие для ФПКП. М.: МИСИ, 1981

[37] Основы теории экстремальных задач для ограниченных аналитических функций и их различных обобщений. Учебное пособие для ФПКП. М.: МИСИ, 1981

[38] Основы теории экстремальных задач для ограниченных аналитических функций с дополнительными условиями. Учебное пособие. М.: МИСИ, 1981

[39] Множества устранимых особенностей аналитических функций. Учебное пособие для слушателей факултета повьшения квалификации. М.: МИСИ 1982

[40] Дополнителшные вопросы теории устранимых особенностей аналитических функций. Учебное пособие для слушателей факултета повышения квалификации. М.: МИСИ, 1982

[41] On a strengthened extremal property of the Carathéodory-Fejér functions // Anal. Math. 1983. V. 9. № 2. P. 99-111

[42] О полных системах в банаховых пространствах // Изв. АН АрмССР. Матем. 1985. Т. 20. № 2. C. 89-111

[43] Представление функций двух переменных суммами $\varphi(x)+\psi(y) / /$ Изв. вузов. Матем. 1985. № 2. C. $66-73$

[44] Two Papers on Extremal Problems in Complex Analysis. Providence, RI: Amer. Math. Soc., 1986. (Amer. Math. Soc. Transl. Ser. 2. V. 129.)

[45] Факторизация однозначных аналитических функций на компактных римановых поверхностях с краем // Зап. научн. семин. ЛОМИ. 1989. Т. 170. № 27. С. 285-313

[46] Об одном методе устранения многозначности аналитических функций // Изв. вузов. Матем. 1990. № 11. C. $64-72$

[47] Теория факторизации однозначных аналитических функций на компактных римановых поверхностях с краем // УМН. 1989. Т. 44. № 4. С. 155-189

[48] Об интерполяционных свойствах внешних функций в суперпозициях Колмогорова // Матем. заметки. 1994. Т. 55. № 4. С. 104-113

[49] Некоторые аппроксимативные свойства линейных суперпорзиций // Изв. вузов. Матем. 1995. № 8. C. $63-73$

[50] Аннулятор линейных суперпозиций // Алгебра и анализ. 1995. Т. 7. № 3. С. 1-42

[51] О существовании равномерного приближения функций нескольких переменных суммами функций меньшего числа переменных // Матем. сб. 1996. Т. 187. № 5. С. 3-14 (совм. с А. Л. Гаркави и В. А. Медведевым)

[52] Best Approximation by Linear Superpositions (Approximate Nomography). Providence, RI: Amer. Math. Soc., 1997. (Transl. Math. Monogr. V. 159.)

[53] Суммы Голубева: теория экстремальных задач типа задачи об аналитической емкости и сопутствующих аппроксимационных процессов // УМН. 1999. Т. 54. № 4. С. 75-142

[54] Some remarks to problems of approximation with prescribed rate // Oper. Theory Adv. Appl. 2000. V. 113. P. 127-133 
[55] 10 лекций по элементарной математике. М.: МГСУ, 2000

[56] Математический анализ. Дифференциальное исчисление функций одной и нескольких переменных. М.: Соврем. гуманит. ун-т, 2000

[57] Аппроксимация элементами клина с учетом величин аппроксимирующих элементов // Изв. вузов. Матем. 2002. № 10. С. 71-84

[58] Соотношения двойственности в теории аналитической емкости // Алгебра и анализ. 2003. T. 15. № 1. C. $3-62$

[59] Аппроксимативные свойства некоторых множеств в пространствах непрерывных функций // Anal. Math. 2003. V. 29. P. 87-105

\section{Публикации о С.Я. Хавинсоне}

[П1] Математика в СССР за сорок лет. 1917-1957. Т. ІІ. М.: ГИФМЛ, 1959. С. 720-721

[П2] Математика в CCCP 1958-1967. T. II. М.: Наука, 1970. С. 1390-1392

[П3] История отечественной математики. 1917-1967. Т. 4, книга 1. Киев: Наукова думка, 1970. С. $219,220,222,306,810,812,813 ;$ T. 4 , книга 2. С. 619

[П4] А. А. Гончар, М. М. Джрббашян, А. Ф. Леонтьев, Г. Ц. Тумаркин, П. Л. Ульянов, Б. В. Шабат. Семен Яковлевич Хавинсон (к 60-летию со дня рождения) // УМН. 1988. T. 43. № 2. C. 173-174

[П5] А. Г. Витушкин, А. А. Гончар, М. В. Самохин, П. Л. Ульянов. Семен Яковлевич Хавинсон (к 70-летию со дня рождения) // УМН. 1997. Т. 52. №3. С. 187-188 